Archives de sciences sociales des religions

112 | octobre-décembre 2000

Âme et corps : conceptions de la personne

\title{
MESSNER (Francis), éd., Les « Sectes » et le droit en
}

France

Paris, P.U.F., 1999, 344 p. (bibliogr.) (coll. « Politique d'aujourd'hui »)

Cyrille Duvert

\section{OpenEdition}

\section{Journals}

Édition électronique

URL : http://journals.openedition.org/assr/20337

DOI : $10.4000 /$ assr.20337

ISSN : $1777-5825$

Éditeur

Éditions de l'EHESS

Édition imprimée

Date de publication : 31 décembre 2000

Pagination : 111-114

ISBN : 2-222-96698-1

ISSN : 0335-5985

\section{Référence électronique}

Cyrille Duvert, «MESSNER (Francis), éd., Les « Sectes » et le droit en France », Archives de sciences sociales des religions [En ligne], 112 | octobre-décembre 2000, document 112.38, mis en ligne le 19 août 2009, consulté le 21 septembre 2020. URL : http://journals.openedition.org/assr/20337 ; DOI https://doi.org/10.4000/assr.20337

Ce document a été généré automatiquement le 21 septembre 2020

(C) Archives de sciences sociales des religions 


\title{
MESSNER (Francis), éd., Les « Sectes » et le droit en France
}

\author{
Paris, P.U.F., 1999, 344 p. (bibliogr.) (coll. « Politique d'aujourd'hui »)
}

\author{
Cyrille Duvert
}

\section{RÉFÉRENCE}

MESSNER (Francis), éd., Les « Sectes » et le droit en France, Paris, P.U.F., 1999, 344 p.

(bibliogr.) (coll. « Politique d'aujourd'hui »)

1 Peut-être fallait-il le refuge de terres concordataires pour réussir le pari lancé par F.M. et les participants à ce colloque organisé à Strasbourg les 13 et 14 juin 1997 : celui de parler d'une question sensible en échappant aux sables mouvants, faits de rumeurs et d'approximations, dans lesquelles s'enlise si souvent le débat sur les sectes, sans pour autant tomber dans l'irénisme naïf et l'empathie par trop indulgente reprochée parfois - injustement - aux sociologues et juristes qui travaillent sur la question.

2 Si l'ouvrage tiré de cette manifestation n'échappe pas toujours au premier de ces travers (les aspects de droit pénal reprennent pour une large part les informations erronées du rapport Gest-Guyard de 1996; la dissolution des Enfants de Dieu en 1978 est présentée comme une sanction administrative "pour racolage et prostitution" alors qu'il s'agissait d'une autodissolution), il n'en constitue pas moins une mine documentaire extrêmement riche (une monographie des principaux mouvements est proposée ainsi qu'une épaisse bibliographie des différentes publications existant en langues française et étrangères) autant qu'un stimulant outil de travail et de réflexion.

Fruit du dialogue entre juristes et sociologues, l'ouvrage dégage un accord sur l'analyse et le diagnostic d'un certain nombre de points. Tous les participants s'accordent d'abord sur l'inutilité d'une loi spécifique sur les sectes. Le catalogue des dispositions de droit pénal (R. Koering-Joulin), de droit de la famille (J. Flauss-Diem), de droit fiscal (G. Siat) ou de droit des associations (E. Sander) fait bien ressortir que, pour le droit, les sectes ne présentent pas de spécificité : seuls comptent les effets et conséquences que 
peut entraîner l'appartenance à un mouvement de ce type et, loin de succomber aux demandes d'interventionnisme accru, particulièrement sensibles en droit de la famille (J. Flauss-Diem), le juge se contente d'agir lorsque ces effets se manifestent, sans attacher d'importance particulière aux choix confessionnels. Pour le droit, la religion est un fait social parmi d'autres sur lequel le juriste n'a en réalité, d'une manière qui décevra peut-être certains, pas grand-chose à dire. Cela est particulièrement vrai du droit public, à propos duquel P.-H. Prélot note que la jurisprudence administrative concernant les sectes est quasiment inexistante (p. 169).

Ce désintérêt relatif pour le fait religieux résulte de la position de neutralité de l'État et partant, du droit, à l'égard de toute croyance, qu'elle soit religieuse ou non. D'où l'inutilité et plus radicalement encore l'impossibilité de toute définition juridique de la secte ou de la religion. Car si le sociologue cherche à définir pour se forger des outils d'analyse, le juriste, lui, le fait pour appliquer un corps de règles spécifiques à la situation ou au phénomène ainsi circonscrit. Le droit français, mais également le droit européen (J. Duffar) assurant l'égalité de toutes les croyances devant la loi, il est impossible, à moins de souhaiter favoriser ouvertement tel ou tel culte, de distinguer en les définissant l'un et l'autre le religieux légitime du religieux illégitime. À cet égard, les auteurs se font pédagogues en montrant bien qu'il ne s'agit pas tant d'une impossibilité matérielle que d'une impossibilité politique: c'est parce que c'est le libéralisme en matière de croyances qui prévaut dans l'espace européen et même occidental (Duffar) que de telles distinctions sont, aujourd'hui, impossibles. Une définition de la secte et en conséquence un régime qui serait spécifiquement applicable à celles-ci seraient néanmoins possibles pourvu qu'ils reposent sur des critères clairs et précis (J.-M. Woehrling). Ce serait néanmoins rompre avec un régime de type libéral et opter pour une limitation de la liberté religieuse, au nom d'une conception normalisatrice de l'activité religieuse, ce qui serait difficilement admissible dans un État qui se veut de droit : " placer la liberté de croire sous surveillance serait donner naissance à une police de la pensée " (Ph. Ségur, p. 144). Il y a donc là un choix politique, et non pas, comme font parfois mine de le croire certains, un simple ajustement technique.

5 L'absence de définition de la secte ne laisse cependant pas les juristes sans outils puisque, on l'a dit, ils appréhendent le phénomène à travers ses effets; et il n'y a qu'un paradoxe apparent à consacrer un ouvrage entier à un objet dont les contours persistent à demeurer flous : la chose ne surprendra ni les sociologues des religions, qui ne s'étonnent plus de travailler sur un objet qu'ils ne définissent pas, ni les juristes qui, pour prendre un exemple tiré d'un autre domaine, ont pu élaborer un droit de la famille sans pour autant définir cette dernière.

6 Les A.A. convergent également pour souligner, en dépit de l'absence de définition juridique de la religion en droit français, le poids. dans les faits, du modèle fourni par l'Église catholique. Les rares dispositions de droit positif destinées à accueillir le fait religieux dans sa spécificité sont en effet toujours, depuis la loi de 1905, calquées sur le modèle d'organisation de cette dernière qui bénéficie alors, de facto, d'avantages qui sont pour l'heure refusés aux sectes ou " Nouveaux Mouvements Religieux ». C'est le cas de la reconnaissance du statut de congrégation religieuse, que seule une communauté bouddhiste est parvenue à obtenir aujourd'hui, du régime d'assurance sociale des ministres du culte, ou de la reconnaissance de la qualité d'association cultuelle, nécessaire pour recevoir les dons et legs dépassant un certain montant. La 
chose est particulièrement flagrante en droit du travail où, note P. Strasser, "l'ensemble des solutions appliquées à la confrontation entre les religions et le Droit du travail ne brillent pas par leur limpidité", renforçant le sentiment que "les relations entre l'institution religieuse et les personnes qui y œuvrent (...) ont bénéficié d'une sorte d'extra-territorialité par rapport au Droit du travail, comme si tout le monde s'accommodait de la solution dans laquelle leurs problèmes, y compris ceux du travail effectué, devaient être réglés "en famille», sans subir en tout cas les contraintes du Droit du travail» (p. 252). Intéressant exemple d'un relatif pluralisme juridique, comme le désignent les sociologues du droit, sous la tutelle indulgente d'un État volontairement en retrait : celui-ci préfère confier aux institutions concernées (en pratique, les quatre cultes antérieurement reconnus), l'organisation de leurs rapports avec ceux de leurs sujets qui décident de leur vouer leur corps et leur âme. Les sectes, qui ne bénéficient évidemment pas de la même légitimité historique et sociale, ne sont pas logées à la même enseigne, et cette distinction de faits se manifeste aussi dans la pratique administrative. Et de rappeler alors le refus, entériné par le Conseil d'État en 1985, de reconnaître la qualité d'association cultuelle aux Témoins de Jéhovah, pour des motifs « dépourvus de précision» (J.-M. Woehrling, p. 74) et de souhaiter, en même tant que l'ouverture d'un débat plus général sur les mutations du paysage religieux depuis la loi de 1905, un réexamen de cette solution. C'est peut-être, notons-le aujourd'hui, la voie dans laquelle désirent s'engager les juges du Palais royal puisqu'ils sont récemment revenus sur leur jurisprudence par une décision du 23 juin 2000 (Ministre de l'Économie c./ Association locale pour le culte des témoins de Jéhovah de Clamecy, in Recueil Dalloz-Sirey 20 juillet 2000, $\mathrm{n}^{\circ} 28$, Informations rapides p. 204).

7 Le constat de cette mutation du paysage socio-religieux français transparaît, en filigrane, dans la plupart des interventions à ce colloque. Partant de ce diagnostic, les uns et les autres font alors diverses propositions. Nombreux sont ceux qui jugent intenable la position officielle d'ignorance par le droit français du fait religieux. Il faut, dit P. Roland, «sortir de l'hypocrisie et de la fiction qui consistent à ne pas donner d'existence juridique à un fait social, non seulement stable, mais présentant des caractères suffisamment spécifiques et distincts pour ne pouvoir se contenter du seul droit commun» (p. 298). Partant, les propositions divergent sur les moyens d'y parvenir, suivant qu'elles ambitionnent de réserver ou non un traitement particulier aux sectes au sein de la question religieuse (J.-M. Woehrling), qu'elles y voient une question intéressant essentiellement la laïcité à la française ou, comme le pense plus largement F.M., un enjeu majeur à venir, celui d'une éventuelle politique européenne des cultes. Au sein des diverses propositions, une faveur semble se dégager à la mise en place d'un système de reconnaissance des cultes qui présenteraient une certaine utilité sociale. Selon des critères précisément définis, certains pourraient ainsi prétendre à des avantages particuliers, tandis que les autres, sans pouvoir prétendre subir une discrimination, auraient la possibilité de choisir entre une simple existence de fait et une existence juridique plus officielle. L'idée, qui rejoint les réflexions développées par J.-P. Costa aux Mélanges Dupuis (L. G. D. J., 1997), permettrait de «tirer l'ensemble du secteur social du « croire » vers le haut, en valorisant les mouvements soucieux de leur intégration et de leur utilité sociale, plutôt que de repousser "vers le bas » les mouvements «à risque » en favorisant ainsi leur marginalisation » (J.-M. Woehrling, p. 89).

8 Que l'on partage ou non les vues et propositions des participants à ce colloque, on ne peut que saluer leur volonté de dépasser l'éternel consensus, quelque peu lénifiant, 
s'établis-sant autour du ni-ni : ni répression accrue par l'adoption d'une législation spécifique, ni indulgence coupable de la part des tribunaux, mais également statu quo sur la question religieuse et laïque. En abordant le problème de front tout en veillant à ne pas se laisser ins-trumentaliser par les acteurs eux-mêmes (J.-P. Willaime), en cherchant à diagnostiquer et analyser les mutations du croire français sans négliger l'inquiétude légitime que peut faire naître le phénomène des sectes ni succomber à la tentation d'une gestion sécuritaire du religieux, en formulant des propositions concrètes, tous répondent au souhait, exprimé par $\mathrm{F}$. Champion et $\mathrm{M}$. Cohen dans ces pages (Arch. $\mathrm{n}^{\circ}$ 96, pp. 5-15) d'une recherche consciente de ses responsabilités sociales. Sur un sujet aussi passionnel, dans lequel se mêlent peurs, angoisses et craintes irrationnelles, le mérite est grand d'avoir su conserver la distance nécessaire à une approche raisonnable et raisonnée de la question, sans pour autant s'enfermer dans la sécheresse bien souvent prêtée aux juristes. En refermant ce livre, on ne peut alors que souscrire à la noble et belle conception du droit que propose P. Roland en conclusion (p. 299) : «La démarche juridique, comme l'avait déjà rappelé Camille Desmoulins en pleine Terreur, est, pour la société, la seule sauvegarde d'une approche raisonnable devant des situations ou des phénomènes qu'elle ne saisit pas et dont elle a peur. Le droit exige, comme chacun le sait, des preuves, un débat contradictoire, le souci de distinguer toutes les situations pour éviter l'amalgame. Il rappelle enfin les principes fondateurs de notre société, ceux-là même qu'elle ne saurait oublier sans se nier. On peut dire que le droit est ici un véritable exercice d'hygiène mentale qu'on peut proposer à tous. C'est en tout cas le seul comportement qui convienne si on adopte le principe de liberté que proclame notre République. » 